\title{
Leitbild des Fachgebietes der Dermatologie und Venerologie
}

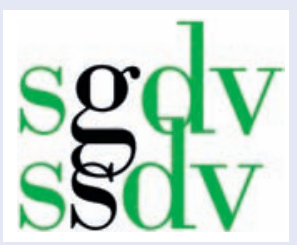

Für die SGDV:

Prof. Peter Itin

Past-Präsident, Basel
Hauterkrankungen sind ausserordentlich häufig und führen oft zu einer erheblichen Beeinträchtigung der Lebensqualität. 20\% der Bevölkerung haben ein therapiebedürftiges Hautleiden und 3,9\% der 80 Millionen ärztlichen Konsultationen in der Schweiz pro Jahr werden durch Hautprobleme verursacht. $7 \%$ aller medizinischen Verordnungen gehen auf das Konto von Dermatologica.

Das Fachgebiet der Dermatologie und Venerologie beinhaltet:

- die Anatomie, die Physiologie, die makroskopische, dermatoskopische und mikroskopische Pathologie der Haut, ihrer Anhangsgebilde und der hautnahen Schleimhäute;

- die venerischen Erkrankungen;

- die allergischen Krankheiten der Haut, einschliesslich des atopischen Syndroms;

- die gutartigen und die bösartigen Tumoren der Haut;

- die peripher-vaskulär bedingten Dermatosen;

- die degenerativen und altersbedingten Hautveränderungen;

- die Photobiologie sowie alle Verfahren der makroskopischen, dermatoskopischen, mikroskopischen und mikrobiologischen Diagnostik;

- alle wissenschaftlich anerkannten Therapien, die Prävention und die Genetik der Hautkrankheiten.

Die Vielfalt des Fachgebietes vereinigt mehr als 2000 verschiedene Diagnosen und deckt sowohl die somatischen als auch die psychosomatischen Affektionen der Haut ab und befasst sich auch mit den psychosozialen und psychosexuellen Aspekten der Dermatosen und der venerischen Krankheiten. Diese Aufzählung zeigt, dass die Dermatologie und Venerologie sehr interdisziplinär ausgerichtet ist, und eine gute Kooperation mit allen Fachrichtungen im Sinne der optimalen Patientenversorgung eine absolute Notwendigkeit ist.

Der Dermatologe beschäftigt sich mit der Erkennung, Therapie und Prävention von obengenannten Haut- und Geschlechtskrankheiten.

Die Schweizerische Gesellschaft für Dermatologie und Venerologie (SGDV) wurde 1913 gegründet, mit dem Ziel, die wissenschaftliche Erforschung und Behandlung der Haut in allen
Bereichen zu fördern. Die SGDV vereinigt heute 391 aktiv tätige Fachärzte.

Die SGDV vertritt die standespolitischen Anliegen und Interessen ihrer Mitglieder, der Gesellschaft und der gesamten Fachdisziplin. Wichtige Ziele der SGDV sind eine strukturierte Weiterbildung mit Vermittlung von Wissen und Fertigkeiten, die im Rahmen einer Facharztprüfung beurteilt werden. Zusätzlich engagiert sich die SGDV für die Fortbildung ihrer Mitglieder und sie führt im Rahmen der Qualitätskontrolle auch eine regelmässige Überprüfung der Fortbildung durch. Die SGDV ist international vernetzt und fördert den globalen Wissensaustausch auf dem Gebiet der Dermatologie und Venerologie.

Die SGDV organisiert zweimal pro Jahr einen nationalen Kongress im Sinne des Wissenstransfers, aber auch mit der Absicht des persönlichen Kontaktes ihrer Mitglieder. Das Gesellschaftsorgan ist die Zeitschrift «Dermatologica Helvetica» und das wissenschaftliche Publikationsorgan die Zeitschrift «Dermatology».

Die Weiterbildung dauert fünf Jahre und ist vollständig fachspezifisch. Daran können maximal je sechs Monate Weiterbildung in Angiologie und Allergologie/klinischer Immunologie angerechnet werden.

Die SGDV hat folgende Ziele:

1. Förderung der Dermatologie und Venerologie in klinischen und wissenschaftlichen Belangen;

2. Förderung des Nachwuchses;

3. Qualitätssicherung im Fachgebiet Dermatologie und Venerologie;

4. Auseinandersetzung mit standespolitischen Fragen;

5. Förderung der nationalen und internationalen Vernetzung;

6. Sicherstellung der dermatologischen Versorgung der Bevölkerung.

Weitere Informationen:

Präsident:

Dr. Thomas Hofer, Wettingen

Vizepräsident:

PD Dr. Stephan Lautenschlager, Zürich

www.derma.ch 